\title{
An Approach to Comparing Multidimensional Geometric Objects
}

\author{
Igor Seleznev ${ }^{1}$, Evgeniy Konopatskiy ${ }^{1}$, Olga Voronova ${ }^{1}$, Oksana Shevchuk ${ }^{1}$ and Andrey \\ Bezditnyi $^{2}$ \\ ${ }^{1}$ Donbas National Academy of Civil Engineering and Architecture, Derzhavina Street, 2, Makeevka, 286123, \\ Ukraine \\ 2 Sevastopol branch of «Plekhanov Russian University of Economics», Vakulenchuk Street, 29, Sevastopol, \\ 299053, Russian Federation
}

\begin{abstract}
The paper proposes an approach to the comparison of multidimensional geometric objects, which is used to assess the variational geometric models of multifactor processes and phenomena obtained using the geometric theory of multidimensional interpolation. The proposed approach consists of two stages, the first of which consists in the discretization of multidimensional geometric objects in the form of a set of discretely given points, and the second is in comparing the obtained discrete point sets using a criterion that is essentially similar to the coefficient of determination. In this case, one of the discrete point sets is taken as a reference for comparison with another point set. For a correct comparison of multidimensional geometric models in the form of point equations, which are reduced to a system of parametric equations, it is necessary to perform interconnection of parameters. A computational experiment was carried out on the example of comparing geometric models of the physical and mechanical properties of fine-grained concrete. It showed the possibility of using the proposed approach for comparing multidimensional geometric objects and the reliability of the results obtained in comparison with scientific visualization methods. On the same example, it was found that for an accurate comparison of the investigated geometric models of the physical and mechanical properties of fine-grained concrete, it is enough to discretize 100 points. A further increase in the set of discrete points of the compared geometric objects has no significant effect on the criterion for assessing their similarity.
\end{abstract}

\section{Keywords}

Geometric object, comparing multidimensional objects, similarity assessment criterion, coefficient of determination, discretization, point sets, 3D model, response surface, point calculus.

\section{Introduction}

During the development of the geometric theory of multidimensional interpolation [1-3], it was found that the geometric models of multifactor processes obtained using multidimensional interpolation are characterized by variability. It is a consequence of the multiplicity of choice of reference lines in the process of developing a geometric process modeling scheme. Along with the variability of geometric models of the same process, the problem arose of choosing the optimal model from the available set of variations. The difficulty of this choice lies in the fact that all possible variations of geometric interpolants fully satisfy the initial experimental and statistical data, but have different curvatures between the interpolation nodal points. The solution to this problem led to the need to compare geometric objects with each other. It turned out that this issue is poorly researched not only in point calculation [4-5]. The authors managed to find a fairly large array of works [6-7] devoted to the

GraphiCon 2021: 31st International Conference on Computer Graphics and Vision, September 27-30, 2021, Nizhny Novgorod, Russia EMAIL: i.v.seleznyov@yandex.ru (I. Seleznev); e.v.konopatskiy@mail.ru (E. Konopatskiy); kornilova.oly@mail.ru(O.Voronova); ks81@ rambler.ru (O. Shevchuk); bezdytniy@gmail.com (A. Bezditnyi)

ORCID: 0000-0002-5491-9827 (I. Seleznev); 0000-0003-4798-7458 (E. Konopatskiy); 0000-0003-3740-5151 (O. Voronova); 0000-00029224-0671 (O. Shevchuk); 0000-0003-0528-9731 (A. Bezditnyi)

(c) (i) (C) 2021 Copyright for this paper by its authors.

Use permitted under Creative Commons License Attribution 4.0 International (CC BY 4.0).

CEUR Workshop Proceedings (CEUR-WS.org) 
development of curve matching tools (maximum information coefficient, Frechet distance, Dynamic Time Warping, etc.), which are used in many applied fields of science and technology, such as time analysis series, speech recognition, signature verification, etc. All these methods are focused on matching one-parameter sets of points - lines and have no generalization to multidimensional space, which could be used to match multi-parameter sets of points. In works [8-9], an approach is proposed to compare three-dimensional geometric objects (human and mannequin bodies) using stochastic methods, using form functions. Many articles are devoted to the comparison of 2D and 3D geometric objects based on various approaches, which include the use of: generate specific distance histograms that define a measure of the geometric similarity of the inspected objects [10], a probabilistic method to evaluate 3D surfaces is presented [11], graph matching technique to measure the distance between these graphs [12], Hausdorff distance (HD) and the accumulated distance difference (ADD) [13], graph similarity into PPM and similarity measurement based on Topological Relationship Distribution (TRD) feature [14], a probability distribution function (PDF) produced from spatial disposition of 3D keypoints, keypoints which are stable on object surface and invariant to pose changes [15], a method of sequential application of global descriptors, allowing the first stage to produce a "rough" screening of obviously different objects, and then to apply more accurate algorithms on a significantly reduced object base [16]. Also, there are a number of review articles devoted to research on the problem of comparing 2D and 3D geometric objects [17]. However, from the works considered, it is not clear whether the proposed method has a generalization to a multidimensional space and whether it can be adapted for use in point calculus.

Traditionally, the method of scientific visualization is used to compare geometric objects overlapping. However, it is only suitable for comparing one- and two-parameter geometric objects. At the same time, even a comparison of two-parameter geometric objects encounters a number of difficulties and the need to use an interactive three-dimensional environment to visualize the comparison results. Comparison of multiparameter geometric objects belonging to a multidimensional space causes a number of practically unrealizable problems associated with the complexity of visualizing geometric objects in multidimensional space. Therefore, it becomes necessary to develop a criterion for assessing the similarity of geometric objects, which could numerically characterize the degree of their coincidence with each other, taking into account the prospective use in multidimensional space.

\section{Evaluation of the similarity of multidimensional geometric objects in point calculus}

One of the features of the point calculus is that all geometric objects in the point calculus are represented by an organized set of points, which are defined using the current point. The current point fills the space with its movement, thereby forming a geometric object. The current parameter is responsible for the movement of the current point, which is an invariant of parallel projection and in most cases varies from 0 to 1 . Continuous change of the current parameter within the specified limits forms a continuous geometric object. However, if you fix a number of values of the current parameter, then you can select a series of fixed points that belong to the modeled geometric object. When there are a lot of such points, they can characterize the geometric model with high accuracy. This feature of the point calculus is proposed to be used to compare several geometric objects, taking into account the generalization to a multidimensional affine space. A multidimensional geometric object in general form is determined by the following point equation:

$$
M=\sum_{i=1}^{m} A_{i} p_{i}(u, v, w, \ldots),
$$

where $A_{i}$ are the initial points, the coordinates of which, in accordance with the geometric theory of multidimensional interpolation [1-3], correspond to the initial experimental and statistical data;

$p_{i}(u, v, w, \ldots)$ are the continuous functions of parameters;

$u, v, w, \ldots$ are the current parameters of the point equation, which in most cases change from 0 to 1 ;

$m$ is the number of origin points. 
Passing from a point equation to a system of the same type of parametric equations, which are projections of a geometric object on the axis of the global coordinate system, we obtain:

$$
\left\{\begin{array}{l}
x=\sum_{i=1}^{m} x_{A_{i}} p_{i}(u, v, w, \ldots) \\
y=\sum_{i=1}^{m} y_{A_{i}} p_{i}(u, v, w, \ldots) \\
z=\sum_{i=1}^{m} z_{A_{i}} p_{i}(u, v, w, \ldots)
\end{array}\right.
$$

It is important to highlight one subtlety. The degree of coincidence of multidimensional geometric objects is determined by the degree of coincidence in all its coordinates. If geometric objects are explicitly specified by equations (for example, a surface $z=f(x, y)$ ), then by setting the same values of the variables $x$ and $y$, it is possible to estimate the degree of coincidence of the surfaces by comparing the corresponding values of the coordinate $z$. In our case, there is a system of parametric equations for which the same values of the parameters can give different coordinates of points that do not correspond to each other. Therefore, for a correct comparison of multidimensional geometric objects in point calculus, it is imperative to correlate the parameters of point equations. In some cases, with a uniform distribution of the initial experimental-statistical data, we can use the special properties of curves passing through predetermined points, obtained on the basis of Bernstein polynomials, to pass from a system of parametric equations to an explicit equation. However, the uniform distribution of the initial experimental and statistical data is a special case and is not always possible in engineering and scientific practice. On the other hand, if there is such a possibility, then when compiling an experiment planning matrix, it is better to use a regular multidimensional network of experimental points, which will greatly facilitate further mathematical processing and analysis of the data obtained.

As noted above, if you continuously change the values of the current parameters in the point equation (1) from 0 to 1, then we get a continuous multidimensional geometric object. But if the values of the parameters are taken with any step, then we get a set of discrete points that belong to this geometric object. For example, for a line that characterizes a one-factor process, using a step of 0.1 for the parameter $u$, we get a set consisting of 11 discrete points. If this step is used for a surface that characterizes a two-factor process, then we get a set consisting of 121 discrete points, etc. Comparing the obtained sets of discrete points, one can estimate their similarity. Of course, the more points a set consists of, the more accurately the degree of similarity can be estimated. But if two geometric objects have very similar shape and position, then for any number of points, the similarity will be very high. For this, one of the statistical criteria can be used, the most popular of which is the coefficient of determination in engineering practice.

The coefficient of determination is usually used to estimate the accuracy of a geometric model obtained using multivariate approximation. It is the ratio of the sum of squared regression residuals to the total variance:

$$
R^{2}=1-\frac{\sum_{i=1}^{m}\left(\hat{y}_{i}-y_{i}\right)^{2}}{\sum_{i=1}^{m}\left(\bar{y}-y_{i}\right)^{2}},
$$

where $\sum_{i=1}^{m}\left(\hat{y}_{i}-y_{i}\right)^{2}$ is the sum of squares of regression residuals, which includes the actual $y_{i}$ and calculated $\hat{y}_{i}$ values of the variable under study;

$\sum_{i=1}^{m}\left(\bar{y}-y_{i}\right)^{2}$ is the total variance;

$\bar{y}$ is the sample mean. 
The coefficient of determination, which is determined by formula (2), can be used as a criterion for assessing the similarity of multidimensional geometric objects. The values of one point, set (selected as a reference) are accepted only as actual values, and another as calculated ones. Similarly, other statistical criteria for assessing similarity can be adapted to compare multidimensional geometric objects.

\section{Computational experiment on the example of comparing geometric models of physical and mechanical properties of fine-grained concrete}

An example of variational geometric modeling of the dependence of the physical and mechanical properties of fine-grained concrete on the composition of the combined aggregate is given, to study the effect of the composition of the combined aggregate in the form of open-hearth slag (OHS), granulated blast furnace slag (GBFS) and burnt rock (BR) on the strength concrete $R_{\mathrm{st}}$. We will use the proposed approach to assess the similarity of the obtained geometric models, the variational geometric schemes of which are shown in Fig. 1.

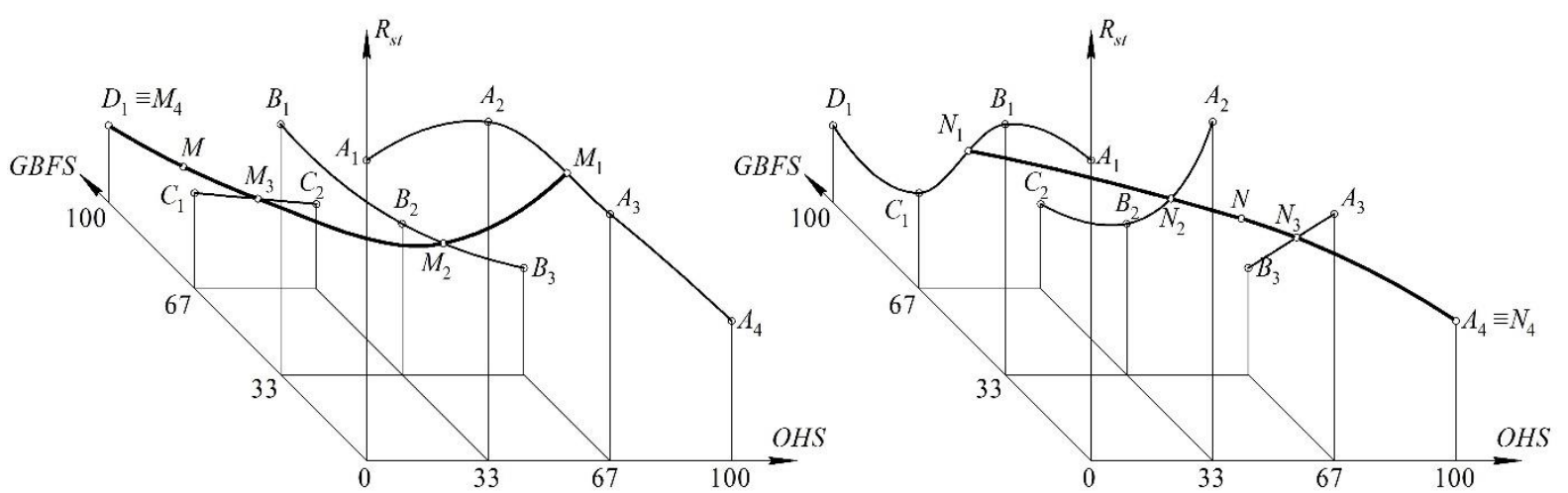

a)

b)

Figure 1: Variational schemes of geometric modeling of the physical and mechanical properties of fine-grained concrete from the composition of the combined aggregate

The first geometric model (Fig. 1a) is described by the following system of parametric equations:

$$
\left\{\begin{array}{l}
\text { OHS }_{1}=100 u_{1}\left(1-v_{1}\right), \\
\text { GBFS }_{1}=100 v_{1}, \\
R_{s t 1}=\left(-99,225 v_{1}^{3}+198,45 v_{1}^{2}-121,275 v_{1}+22,05\right) u_{1}^{3}+\left(247,05 v_{1}^{3}-480,6 v_{1}^{2}+279,45 v_{1}-45,9\right) u_{1}^{2}+ \\
+\left(-184,725 v_{1}^{3}+341,1 v_{1}^{2}-177,625 v_{1}+21,25\right) u_{1}+37,35 v_{1}^{3}-58,05 v_{1}^{2}+16,4 v_{1}+6,4 .
\end{array}\right.
$$

The first geometric model (Fig. 1b) is described by a similar system of parametric equations:

$$
\left\{\begin{array}{l}
\text { OHS }_{2}=100 u_{2}, \\
\operatorname{GBFS}_{2}=100 v_{2}\left(1-u_{2}\right), \\
R_{s t 2}=\left(-168,075 v_{2}^{3}+350,325 v_{2}^{2}-204,75 v_{2}+22,05\right) u_{2}^{3}+\left(336,15 v_{2}^{3}-670,95 v_{2}^{2}+382,95 v_{2}-45,9\right) u_{2}^{2}+ \\
+\left(-205,425 v_{2}^{3}+378,675 v_{2}^{2}-194,6 v_{2}+21,25\right) u_{2}+37,35 v_{2}^{3}-58,05 v_{2}^{2}+16,4 v_{2}+6,4 .
\end{array}\right.
$$

As a result of an analytical comparison of these two geometric models, it was found that both response surfaces pass through 10 initial points and can be considered reliable simulation results. But a comparison of the same models by the method of scientific visualization showed that they are quite close, but differ from each other in the zones highlighted in red (Fig. 2).

Taking into account the peculiarities of geometric schemes for modeling the physical and mechanical properties of fine-grained concrete in the form of two-parameter response surfaces (Fig. 1), which in the plan have the shape of a triangle, it is necessary to correlate the parameters of the systems of parametric equations: 


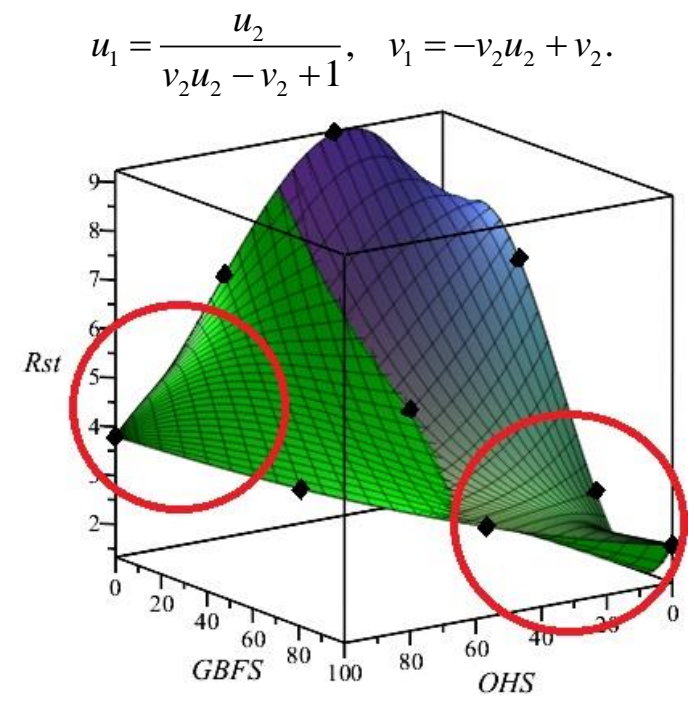

Figure 2: Comparison of variational geometric models by the superposition method

As a result, the system of parametric equations of the first geometric model (Fig.1a) takes the following form:

$$
\left\{\begin{array}{l}
\text { OHS }_{1}=100 u_{2}, \\
\text { GBFS }_{1}=100 v_{2}\left(1-u_{2}\right), \\
R_{s t 1}=\frac{1}{\left(u_{2} v_{2}-v_{2}+1\right)^{3}}\left(-37,35\left(u_{2}-1\right)^{6} v_{2}^{6}+\right. \\
+\left(184,725 u_{2}^{6}-1093,725 u_{2}^{5}+2697,75 u_{2}^{4}-3548,25 u_{2}^{3}+2624,625 u_{2}^{2}-1035,225 u_{2}+170,1\right) v_{2}^{5}+ \\
+\left(-247,05 u_{2}^{6}+1698,75 u_{2}^{5}-4627,1 u_{2}^{4}+6461,9 u_{2}^{3}-4904,85 u_{2}^{2}+1920,95 u_{2}-302,6\right) v_{2}^{4}+ \\
+\left(99,225 u_{2}^{6}-1025,325 u_{2}^{5}+3525,175 u_{2}^{4}-5670,125 u_{2}^{3}+4624,2 u_{2}^{2}-1807,45 u_{2}+254,3\right) v_{2}^{3}+ \\
+\left(198,45 u_{2}^{5}-1156,95 u_{2}^{4}+2436,15 u_{2}^{3}-2283,3 u_{2}^{2}+893,7 u_{2}-88,05\right) v_{2}^{2}+ \\
\left.+\left(121,275 u_{2}^{4}-446,625 u_{2}^{3}+545,475 u_{2}^{2}-217,325 u_{2}-2,8\right) v_{2}+22,05 u_{2}^{3}-45,9 u_{2}^{2}+21,25 u_{2}+6,4\right) .
\end{array}\right.
$$

Next, we will investigate the effect of the discretization of geometric objects on the criterion for assessing similarity. To do this, we will calculate the criterion for assessing the similarity of geometric objects for different sizes of a network of discrete points belonging to the modeled geometric object. The research results are shown in Fig. 3.

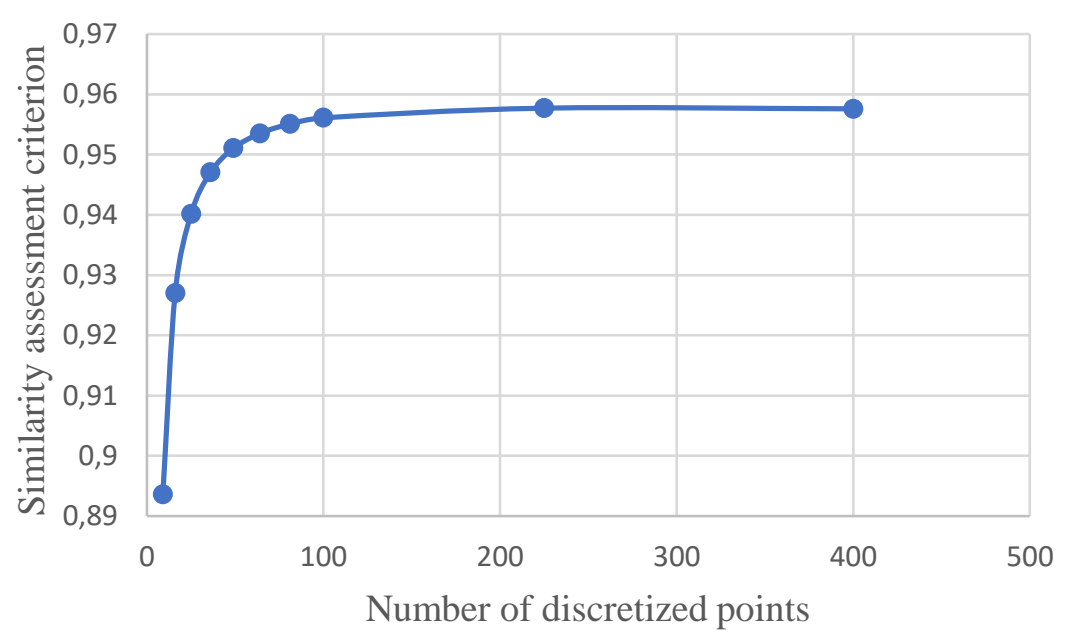

Figure 3: Analysis of the influence of discretization points on the criterion for assessing the similarity of geometric objects 
As can be seen from Fig. 3, the high level of similarity of geometric objects obtained by the method of superimposing response surfaces is fully confirmed. It should be noted that for a specific example, after 100 sampled points, the values of the similarity assessment criterion are aligned and no longer significantly changes, remaining within $R^{2}=0,956$.

\section{Conclusion}

The criterion for assessing the similarity of multidimensional geometric objects, akin to the coefficient of determination from regression analysis, is, on the one hand, a universal tool for comparing multidimensional geometric objects, on the other hand, it has all the disadvantages inherent in the coefficient of determination. For example, the coefficient of determination provides for comparison with the averaged value of the reference variable; therefore, the accuracy of its estimation decreases with a significant scatter of the values of the variable taken as the reference one. And when it is generalized to a multidimensional space, if there is a significant difference in the order of values on each numerical axis of the data, it can give unsatisfactory results. This leads to the need for additional research to develop a criterion for assessing the similarity of geometric objects more adapted to the multidimensional space (for example, it can be one of the statistical criteria: the chi-square test, the Kolmogorov-Smirnov test, the Cramer-Mises test, the Bhattachary test, the Kullback divergence Leibler, Jensen-Shannon divergence, Hamman coefficient, Jackard coefficient, etc.). At the same time, the proposed approach, which is based on the discretization of geometric objects for their comparison both explicitly and parametrically, regardless of the choice of the evaluation criterion, can find wide application in scientific research in various fields of science and technology.

The proposed approach to comparing multidimensional geometric objects based on the similarity criterion, akin to the coefficient of determination from regression analysis, can find a worthy place in a number of existing and innovative approaches not only in multidimensional geometry, but also in decision making theory. If we consider a multiparameter geometric object of a multidimensional space as a graphic display of a multi-factor process, then the proposed one can be used to compare many processes and phenomena in science and technology. It can also be an effective tool for comparing the reference (exact) solution with the numerical solution of partial differential equations by approximating the desired solution by geometric objects with predetermined differential properties [18-19]. Another area of practical application of the proposed method can be a comparison of geometric bodies obtained in point calculus [20].

\section{References}

[1] E.V. Konopatskiy, A.A. Bezditnyi, Geometric modeling of multifactor processes and phenomena by the multidimensional parabolic interpolation method, IoP conference series: Journal of Physics: Conf. Series 1441 (2020), 012063. doi: 10.1088/1742-6596/1441/1/012063.

[2] E.V. Konopatskiy, A.A. Bezditnyi, Application of mixed geometric interpolants for modeling the strength characteristics of steel fiber concrete, IoP conference series: Journal of Physics: Conf. Series 1546 (2020), 012037. doi: 10.1088/1742-6596/1546/1/012037.

[3] E.V. Konopatskiy, A.I. Bumaga, A.A. Bezditnyi, Geometric approach to finding the best possible solutions based on composition optimization of the mixed aggregate of fine-grained concrete, IoP conference series: Materials Science and Engineering: Conf. Series 962 (2020), 032031. doi: 10.1088/1757-899X/962/3/032031.

[4] I.G. Balyuba, Constructive geometry of manifolds in point calculus, Kiev, 1995.

[5] I.G. Balyuba, A.V. Naydysh, Point calculus, Melitopol, 2015.

[6] Meinard Müller, Dynamic Time Warping. Dynamic Time Warping. In: Information Retrieval for Music and Motion, Springer, Berlin, Heidelberg, 2007. doi: 10.1007/978-3-540-74048-3_4.

[7] A. Efrat, S. Venkatasubramanian, Q. Fan, Curve matching, time warping, and light fields: New algorithms for computing similarity between curves, J. Mathematic Imaging and Vision, 2007. http://citeseerx.ist.psu.edu/viewdoc/download?doi=10.1.1.118.5078\&rep=rep1\&type=pdf. 
[8] S.N. Grudinin, Comparison of three-dimensional objects. Similarity assessment criteria, A young scientist, Volume 1, No. 5(28), 2011, pp. 42-44.

[9] S.N. Grudinin, V.D. Frolovsky, Comparison methods for complex geometric objects, In collection: Science of technology innovation. Materials of the All-Russian Scientific Conference of Young Scientists, Novosibirsk State Technical University, 2013, pp. 189-192.

[10] M. Novotni, R. Klein, A geometric approach to 3D object comparison, Paper presented at the Proceedings - International Conference on Shape Modeling and Applications, SMI 2001, pp. 167175. doi: 10.1109/SMA.2001.923387.

[11] J. Pérez, J. Guardiola, A. J. Perez, J. Perez-Cortes, Probabilistic evaluation of 3d surfaces using statistical shape models (ssm), Sensors (Switzerland), Vol. 20, No. 22, 6554, 2020. doi: $10.3390 / \mathrm{s} 20226554$.

[12] K. Madi, E. Paquet, H. Kheddouci, New graph distance for deformable 3D objects recognition based on triangle-stars decomposition, Pattern Recognition, Vol. 90, 2019, pp. 297-307. doi: 10.1016/j.patcog.2019.01.040.

[13] P. Peltola, J. Xiao, T. Moore, A. R. Jiménez, F. Seco, GNSS trajectory anomaly detection using similarity comparison methods for pedestrian navigation, Sensors (Switzerland), Vol. 18, No. 9, 2018. doi: 10.3390/s18093165.

[14] R. Wen, W. Tang, Z. Su, Measuring 3D process plant model similarity based on topological relationship distribution, Computer-Aided Design and Applications, Vol. 14, No. 4, pp. 422-435, 2017. doi: $10.1080 / 16864360.2016 .1257185$.

[15] V.K. Ghorpade, P. Checchin, L. Malaterre, L. Trassoudaine, 3D shape representation with spatial probabilistic distribution of intrinsic shape keypoints, Eurasip Journal on Advances in Signal Processing, Vol. 2017, Issue1, 2017. doi: 10.1186/s13634-017-0483-y.

[16] A.V. Pristupa, I.L. Lapatin, A.V. Zamyatin, 3D object identification based on global shape descriptors, Tomsk State University Journal of Control and Computer Science, No. 48, pp. 57-66, 2019. doi: 10.17223/19988605/48/7.

[17] G. Lara López, A. Peña Pérez Negrón, A. De Antonio Jiménez, J. Ramírez Rodríguez, R. Imbert Paredes, Comparative analysis of shape descriptors for 3D objects, Multimedia Tools and Applications, Vol. 76, Issue 5, pp. 6993-7040, 2017. doi: 10.1007/s11042-016-3330-5.

[18] E.V. Konopatskiy, O.S. Voronova, O.A. Shevchuk, A.A. Bezditnyi, About one method of numeral decision of differential equalizations in partials using geometric interpolants, CEUR Workshop Proceedings, Volume 2763, 2020, pp. 213-219. doi: 10.30987/conferencearticle_5fce27708eb353.92843700.

[19] E.V. Konopatskiy, A.A. Bezditnyi, O.A. Shevchuk, Modeling geometric varieties with given differential characteristics and its application, CEUR Workshop Proceedings, Volume 2744, 2020. doi: 10.51130/graphicon-2020-2-4-31.

[20] E.V. Konopatskiy, A.A. Bezditnyi, M.V. Lagunova, A.V. Naidysh, Principles of solid modelling in point calculus, IoP conference series: Journal of Physics: Conf. Series 1901 (2021), 012063. doi: 10.1088/1742-6596/1901/1/012063. 